\title{
Patrones de actividad del venado cola blanca en el noreste de México
}

\author{
Sonia Gallina ${ }^{1 *}$ y Joaquin Bello Gutierrez ${ }^{2+}$
}

Introduction: Animals' requirements and their complex interactions with their environment result in different activity patterns that reflect adaptations to daily, seasonal and annual variations in their surroundings. These adaptations may differ among individuals as a function of age, sex, physiological state, time of day, season and weather. Behavior is important because deer tend to voluntarily avoid unpleasant environmental circumstances, such as extreme heat or cold, and wind. Most animals divide their time between two fundamentally different types of behavior: activity and rest. All deer species have a unique way of spending their time and energy to maximize their fitness. Studies of behavioral thermoregulation in ungulates report a reduction in activity and an increase in rest in ruminant species on days when the temperature is high.

This suggests that deer are bedding during the day and foraging at night when the temperature is lower. In northeastern desert scrub in Mexico, the activities of white-tailed deer were documented over four years to establish whether activity and behavior patterns change with variations in the environment as a result of the quantity and distribution of annual precipitation.

Methods: This research was carried out at Rancho San Francisco $\left(27^{\circ} 19^{\prime} 36^{\prime \prime}-27^{\circ} 22^{\prime} 24^{\prime \prime} \mathrm{N}, 100^{\circ} 36^{\prime}\right.$ $23^{\prime \prime}-100^{\circ} 39^{\prime} 01^{\prime \prime} \mathrm{W}: 1,000 \mathrm{ha}$ ) located in northeastern Mexico. Climate is semiarid with $400 \mathrm{~mm}$ average annual rainfall. From September 1994 to November 1997 a total of 14 females and 9 males were captured, and each was fitted with a radio collar with an activity sensor. Deer location was determined with TR-4 receivers, portable type $\mathrm{H}$ and fixed 4-element parallel Yagi, null peak type antennas. Animal location was determined simultaneously by two people every hour over 24 hour cycles, with 2-3 monthly cycles over four years. Each time a deer was located its main behavior pattern was noted based on the number of beeps emitted by the tracking equipment over 5 minutes (bedding, moving, feeding).

Results: A total of 243 cycles for females and 170 cycles for males were obtained and analyzed. Deer generally spent more than $12 \mathrm{~h}$ bedding, and females were more active than males. There were significant differences in some behavior patterns of the deer for some years and between sexes, though a crepuscular pattern was found for allfour years. There was a significant difference in bedding for females between 1995 and $1997(P=0.013)$, and for males 1995 between and $1998(P=0.058)$. Between sexes, there were significant differences in activity cycles for $1995(P=0.012)$ and $1998(P=0.058)$, and significant differences between sexes in moving behavior during $1998(P=0.012)$.

There was a strong correlation between annual precipitation and some variables by sex (Table 3): females and males responded differently. Females had high positive values for feeding $(r=0.97)$, but negative values for movement $(r=-0.085)$, indicating that they spent more time feeding and less time moving in years with more rainfall and thus more food available. In contrast, males $(r=-0.92)$ rest more in years with less precipitation, or more pronounced drought conditions.

Discussion and Conclusions: We found females were more active than males though for both sexes the annual pattern was crepuscular. Females increased foraging time from $10 \%$ (1995) to $20 \%$ (1998) when the rains were delayed in 1998 and almost all their fawns died. Studies of behavior in ungulate browsers have

\footnotetext{
'Red de Biología y Conservación de Vertebrados, Instituto de Ecología, A.C. Carretera Antigua a Coatepec \#351, El Haya, Xalapa 91070, Veracruz, México. E-mail: sonia.gallina@inecol.mx (SG)

${ }^{2+}$ División Académica de Ciencias Biológicas. Universidad Juárez Autónoma de Tabasco. Km. 0.5 carretera VillahermosaCárdenas. Entronque a Bosques de Saloya, Villahermosa 86039, Tabasco, México.

${ }^{*}$ Corresponding author
} 
shown that animals reduced their activity and increased bedding time during high daytime temperatures suggesting that deer rest during the hottest hours of the day, and forage at night when it is cooler. Some authors found a significant negative correlation between the periods the deer were active throughout the day and temperature. We conclude that white-tailed deer have a bimodal activity pattern and are most active at dawn and dusk, though this may change with environmental conditions. Some years there are significant differences in their $24 \mathrm{~h}$ activity cycle, indicating their adaptability to changing environmental factors in semiarid conditions, such as forage availability as determined by the quantity of rain.

Key words: bedding, behavior patterns, desert scrub, feeding, moving, radiotelemetry

Los requerimientos de los animales y sus interacciones con el medio ambiente dan como resultado patrones de actividad que son una adaptación a las variaciones diarias y estacionales, y que pueden diferir en cada individuo con la edad, el sexo, el estado fisiológico, la hora del día, la estación y las condiciones climáticas. Con el objeto de conocer el patrón de actividad del venado cola blanca en un matorral xerófilo, de acuerdo al sexo y el año, se presentan los resultados de cuatro años (1995-1998) de estudio en el noreste de México. Utilizando radiotelemetría, siguiendo 14 hembras y 9 machos, durante 2-3 ciclos de 24 h mensualmente, para registrar su patrón de actividades separando las tres pautas importantes (echado, moviéndose o alimentándose). Un total de 243 ciclos para hembras y 170 ciclos para machos fueron obtenidos y analizados. Los venados dedican aproximadamente más de $12 \mathrm{~h}$ diarias a estar echados y las hembras son más activas que los machos. Además, hubo diferencias significativas entre ciertas pautas de comportamiento en algunos años y entre sexos, pero mantienen un patrón de actividad crepuscular. También se encontró que hembras y machos responden de manera diferente a la precipitación: las hembras dedicaron más tiempo a alimentarse y se mueven menos en años con mayor precipitación, y que los machos descansan más en años con menor precipitación o condiciones de sequía más pronunciada.

Palabras clave: alimentarse, desplazarse, echado, matorral xerófilo, pautas de comportamiento, radiotelemetría.

El comportamiento de cualquier animal dependerá de las condiciones intrínsecas del individuo (estado fisiológico, sexo, edad) y de las condiciones extrínsecas como el tipo de vegetación, cantidad y calidad de las plantas disponibles como forraje, cobertura de protección, de la disponibilidad de agua libre, de las características de temperatura, humedad y precipitación del ambiente (Ockenfels y Bissonette 1984; Verme y Ullrey 1984; Beier y McCullough 1990; Ockenfels et al. 1991; Kroll 1992). De las necesidades o requerimientos de los animales y de las complejas interacciones con el medio ambiente, resultan patrones de actividad propios que no son sino una adaptación a las variaciones diarias y estacionales (Lariviere et al. 1994), y que pueden diferir entre individuos de acuerdo con la edad, el sexo, el estado fisiológico, la hora del día, la estación y las condiciones climáticas. Los cambios en los patrones de actividad pueden estar influenciados por factores externos como la temperatura, la presión atmosférica, ciclos de luz y obscuridad, fases lunares y depredadores (Beier y McCullough 1990; Kamler et al. 2007). Se encontró que el metabolismo más bajo en el venado cola blanca ocurre 
en invierno y el más alto en verano, siendo este ritmo una adaptación para conservar energía, la necesidad de recursos forrajeros es menor cuando los recursos disponibles son reducidos (Moen 1978). El comportamiento es importante porque voluntariamente el venado en general, puede evitar circunstancias desagradables del ambiente, como el intenso calor o el frío y los vientos (Wallmo 1981). La mayoría de los animales dividen su tiempo en dos conductas fundamentalmente diferentes: actividad y descanso. Todas las especies tienen un modo único de distribuir su tiempo y energía para maximizar su adecuación.

La disponibilidad de alimento para los herbívoros no cambia en el curso del día, así que los patrones son gobernados por condiciones ambientales, presión de depredación y habilidades digestivas tales como la tasa metabólica (Halle y Stenseth 2000). Las habilidades digestivas son particularmente importantes en rumiantes, cuyo sistema digestivo requiere de períodos regulares de descanso para funcionar apropiadamente. El tiempo destinado a los períodos de descanso son dependientes de la masa corporal o tamaño del cuerpo en rumiantes, siendo los animales pequeños los que generalmente requieren más descanso que los de mayor talla (duToit y Yetman 2005).

$\mathrm{Al}$ analizar los trabajos realizados con diferentes especies de venados se encuentran distintos patrones de actividad. Por ejemplo, Eberhardt et al. (1984) señalan que el venado bura Odocoileus hemionus presentaba un patrón crepuscular, aunque Webb et al. (2013) demuestran que las hembras de esta especie sí exhibieron patrones de movimiento crepuscular (con puntos máximos entre 06:00 y 18:00 h) durante la estación cálida, pero en cambio durante la estación de clima fresco exhibieron un patrón de movimiento diurno (con puntos máximos entre 06:00 y 15:00 h); el venado de las Pampas (Ozotoceros bezoarticus) en el Parque Nacional Emas y en los Cerrados (savana Brasileña) de Brasília, tienen periodos de actividad tanto diurnos como nocturnos (Leeuwenberg et al. 1997; Rodrigues y Monteiro-Filho 2000). Un estudio, por Rivero et al. (2005) realizado con dos especies simpátricas de temazate en Bolivia, mostró que los temazates rojos (Mazama americana) fueron más activos durante la puesta del sol hasta el amanecer (18:00 a 06:00 h), mientras que el temazate gris (M. gouazoubira) lo fue en las mañanas (05:00 a 10:00).

Los estudios sobre termorregulación conductual en ungulados han mostrado una reducción en la actividad y un incremento en el descanso en especies rumiantes ramoneadoras en días con elevada temperatura (du Toit y Yetman 2005). Esto sugiere que los venados descansan durante el día y llevan a cabo su actividad de forrajeo en la noche cuando las temperaturas son menores. Esto mismo encuentran otros autores (Drolet 1976, Cederlund 1989, Beier y McCullough 1990, Leeuwenberg et al. 1997, Webb et al. 2013) señalando que sobre todo en venados cola blanca son factores externos similares los que gobiernan los patrones circadianos de actividad como el fotoperíodo, termorregulación y la depredación. Los venados reducen su actividad durante el periodo del día con mayor estrés térmico.

Estos cambios conductuales se han estudiado también con el venado cola blanca texano en el matorral xerófilo del Noreste de México (Corona-Zárate 1999; Gallina et al. 1998, 2003, 2005; Bello et al. 2001a, 2001b, 2003a, 2003b, 2004). En este trabajo la pregunta a responder es si los venados modifican su patrón conductual dependiendo de las condiciones ambientales, es decir, si la precipitación que difiere cada año, sobre 
todo en zonas áridas, influye en su comportamiento y si los sexos responden de igual manera debido a sus distintos requerimientos energéticos. El objetivo de este estudio fue comparar la conducta del venado cola blanca entre sexos y años. A diferencia de otros estudios, en este caso se están tomando en cuenta las diferentes pautas de comportamiento (echado, desplazarse y alimentarse). Se buscó conocer si existen cambios conductuales, tomando en consideración la diferencia en la cantidad y distribución de la precipitación de los diferentes años. La precipitación ocasiona cambios ambientales, sobre todo en la disponibilidad de alimento, lo cual puede repercutir en el patrón conductual de los individuos.

Área de estudio. Este estudio se llevó a cabo en el Rancho San Francisco (27 $19^{\prime} 36^{\prime \prime}$ y Métodos - $27^{\circ} 22^{\prime} 24^{\prime \prime} \mathrm{N}, 100^{\circ} 36^{\prime} 23^{\prime \prime}$ - $100^{\circ} 39^{\prime} 01^{\prime \prime} \mathrm{W}$ ) localizado entre los municipios de Lampazos, Nuevo León y Progreso, Coahuila, en el Noreste de México. El rancho tiene una superficie de 1,000 ha cercadas con malla venadera y tiene un manejo del agua intensivo ( 3 presones artificiales construidos para captar agua de lluvia y 32 bebederos de cemento distribuidos en todo el rancho). La altitud es de 430 m; el clima es semiárido con una temperatura media anual de $21^{\circ} \mathrm{C}$, aunque la máxima puede exceder los 40 ${ }^{\circ} \mathrm{C}$. La precipitación promedio anual es menor a $400 \mathrm{~mm}$, pero presenta una notable variación mensual y anual. El climograma de los años 1995 a 1998 que se presenta en la Figura 1 fue obtenido de la estación meteorológica Venustiano Carranza, que se localiza a 10 km del Rancho San Francisco, Nuevo León, México. La precipitación anual fue de 135.5 mm en 1995, 285.5 en 1996, 368 mm en 1997 y 318 mm en 1998. La vegetación es matorral xerófilo (Briones 1984).

Desde septiembre a noviembre de1994, 1995, 1996 y 1997 se capturaron un total de 14 hembras y 9 machos. A cada venado se le colocó un radiocollar con frecuencia distinta que tienen sensor de actividad (Telonics, Inc. Meza, Arizona), y fueron localizados con receptores TR-4, antenas portátiles tipo $\mathrm{H}$ y fijas tipo Yagui paralelas con cuatro elementos, de pico nulo. Las localizaciones de los animales fueron realizadas de manera simultánea por dos personas en sitios fijos para poder triangular y determinar el lugar de mayor probabilidad donde se encontraba el animal, cada hora durante ciclos de 24 h, por dos a tres ciclos mensuales al año, y en cada hora se determinó la pauta de comportamiento de cada individuo de venado. Las pautas fueron identificadas mediante el conteo de las pulsaciones que emitían los collares, durante cinco minutos por individuo. Se consideraron, tres pautas con una confiabilidad del $90 \%$, de acuerdo a Mandujano et al. (1996): echado (con una frecuencia de 50-55 pulsaciones por minuto), moviéndose (5669) y alimentándose (70-87 pulsaciones por minuto). Considerando los ciclos de $24 \mathrm{~h}$ se obtuvo la frecuencia por pauta juntando la información de todas las hembras localizadas en cada año para poder comparar los datos con los de los machos, con el objeto de determinar si existe un patrón de comportamiento similar y si hay diferencias en las respuestas a las condiciones ambientales. Se usaron pruebas de Kruskall-Wallis para comparar la frecuencia de las pautas en ciclos de $24 \mathrm{~h}$ de machos y hembras por separado entre años. Con una prueba post-hoc de Comparaciones Múltiples de Mann-Whitney con el Programa PAST (Hammer et al. 2001) se buscaron diferencias significativas de los patrones de conducta por sexo y año. La prueba de F se usó para comparar entre sexos 
(machos y hembras) cada pauta por separado (echado, desplazarse o alimentarse), para conocer si las respuestas conductuales difieren y si alguno de los sexos es más sensible a los cambios provocados por las distintas condiciones ambientales. Los coeficientes de correlación permitieron correlacionar la frecuencia de cada pauta por sexo por año y la precipitación anual para ver si había un efecto sobre el comportamiento de los venados (Zar 1984).

Figura 1. Climograma de los años 1995 a 1998 de la estación meteorológica Venustiano Carranza que se localiza a $10 \mathrm{~km}$ del Rancho San Francisco, Nuevo León, México, presentando en el eje $Y$ de la izquierda la precipitación ( $\mathrm{P}$ en $\mathrm{mm}$ ) $y$ en el de la derecha la temperatura máxima (TM en ${ }^{\circ} \mathrm{C}$ ).

\section{CLIMOGRAMA}

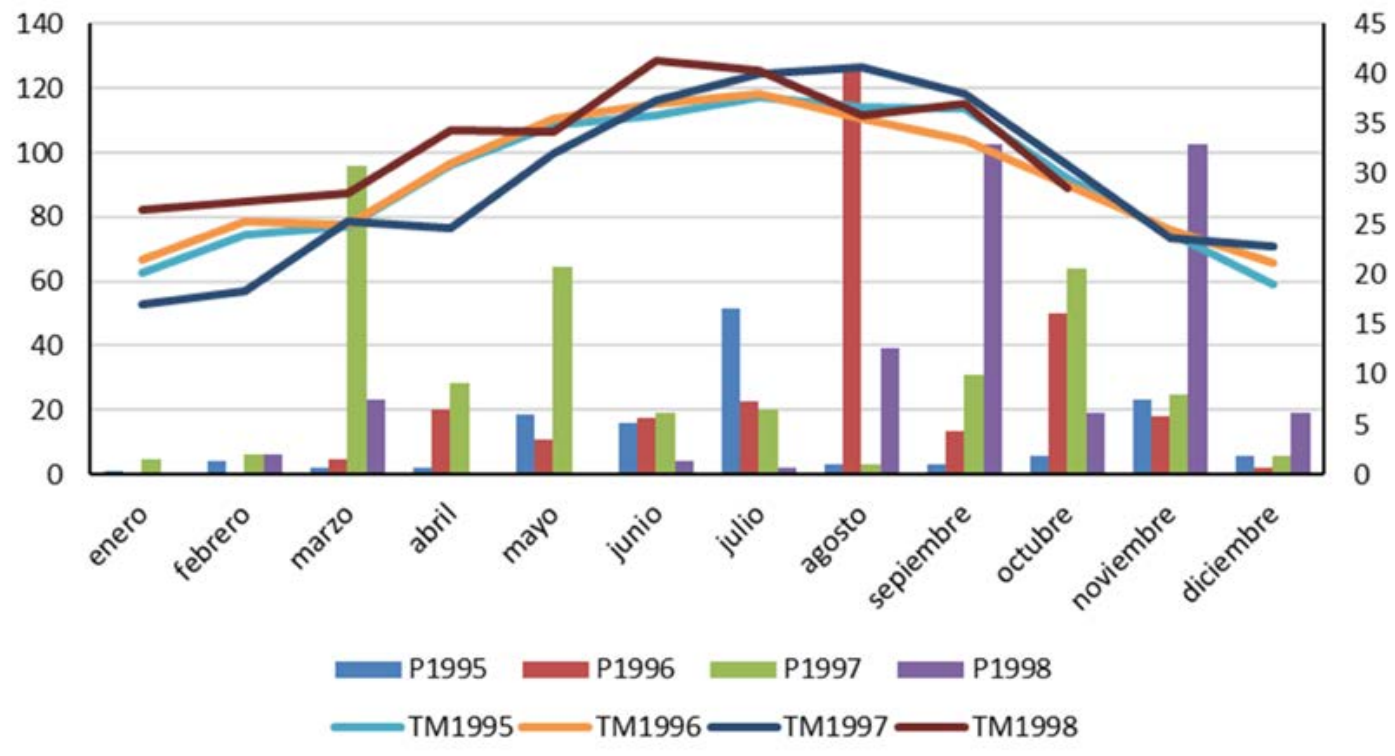

\section{Resultados}

Un total de 243 ciclos para hembras y 170 ciclos para machos de $24 \mathrm{~h}$ fueron obtenidos durante los cuatro años de estudio: 3,715 horas efectivas fueron utilizadas para los análisis con hembras y 2,587 horas para machos (Tabla 1). El porcentaje total dedicado a cada pauta por hembras y machos, en los diferentes años revela que los machos en general dedican una mayor proporción de su tiempo a estar echados a diferencia de las hembras que ocupan más tiempo a desplazarse y alimentarse. El patrón conductual cambia con los años (Fig. 2).

Se obtuvo el ciclo de 24 horas, separando cada pauta para conocer si siguen un patrón crepuscular de actividad como se ha encontrado en otros estudios y en que horario presentan con mayor frecuencia las diferentes conductas (Fig. 3-5) y si esto cambia con los años. Los resultados de la Prueba de Kruskal-Wallis fueron no significativos: para la pauta echado $\mathrm{H}=4.903, P=0.672$; desplazarse $\mathrm{H}=.907, P=0.3407$ y alimentarse $\mathrm{H}$ $=11.1, P=0.134$, pero al hacer las Comparaciones Múltiples de Mann-Whitney sí se encontraron algunas diferencias entre años y sexos en algunas de las pautas:

Echado: No se encontró diferencias significativas temporalmente en las hembras. En 1995 se muestran dos picos: uno a las 04:00 y otro a las 20:00 h, mientras que en 1997 no son tan notorios los picos de descanso y ocurren a las 05:00 y entre las 20:00 y 22:00 h (Fig. 3a). En los machos, los análisis de comparación múltiple tampoco muestran una diferencia en este comportamiento. Las horas que pasaron más tiempo echados en 
1998 fueron a las 03:00 y entre 10:00 y 11:00 h, mientras que en 1995 esta actividad fue entre las 12:00 y 13:00 h (Fig. 3). Se encontró diferencias significativas en los ciclos de actividad entre los sexos en $1995(P=0.012)$ y en $1998(P=0.058)$, que fueron años contrastantes en cuanto a la precipitación, como se aprecia en el climograma (Fig. 1).

\begin{tabular}{lllllll}
\hline Año & $\begin{array}{l}\text { No. Ind. } \\
\text { Hembras }\end{array}$ & $\begin{array}{l}\text { No. Ind. } \\
\text { Machos }\end{array}$ & $\begin{array}{l}\text { Total Horas } \\
\text { Hembras }\end{array}$ & $\begin{array}{l}\text { Total Horas } \\
\text { Machos }\end{array}$ & $\begin{array}{l}\text { Total Ciclos de 24 h } \\
\text { Hembras }\end{array}$ & $\begin{array}{l}\text { Total Ciclos de 24 } \\
\text { h Machos }\end{array}$ \\
\hline 1995 & 3 & 2 & 908 & 549 & 62 & 42 \\
1996 & 3 & 3 & 918 & 502 & 70 & 47 \\
1997 & 6 & 5 & 1061 & 1018 & 61 & 48 \\
1998 & 6 & 4 & 828 & 518 & 50 & 33 \\
total & & & 3,715 & 2,587 & 243 & 170 \\
\hline
\end{tabular}

Tabla 1. Tamaño de muestra de los venados cola blanca tomando en cuenta sexo y año, en el Rancho San Francisco, Nuevo León, México.

Desplazarse: Se encontraron diferencias significativas en la pauta de desplazamiento en los diferentes años entre las hembras en los años 1995 y $1998(P=0.019)$, apreciándose un pico a las 17:00 en 1995 y a las 07:00 h en el año 1996. De las 07:00 a las 09:00 y de las 17:00 a las 19:00 las hembras tienden más a moverse, siguiendo entonces un patrón bimodal (Fig. 4). En cambio no se encontraron diferencias significativas en los machos en los diferentes años en esta actividad, y en general se aprecia que se mueven más entre las 06.00 y 09:00 de la mañana (Fig. 4). Entre sexos las diferencias significativas se encontraron únicamente en el año $1998(P=0.025)$.

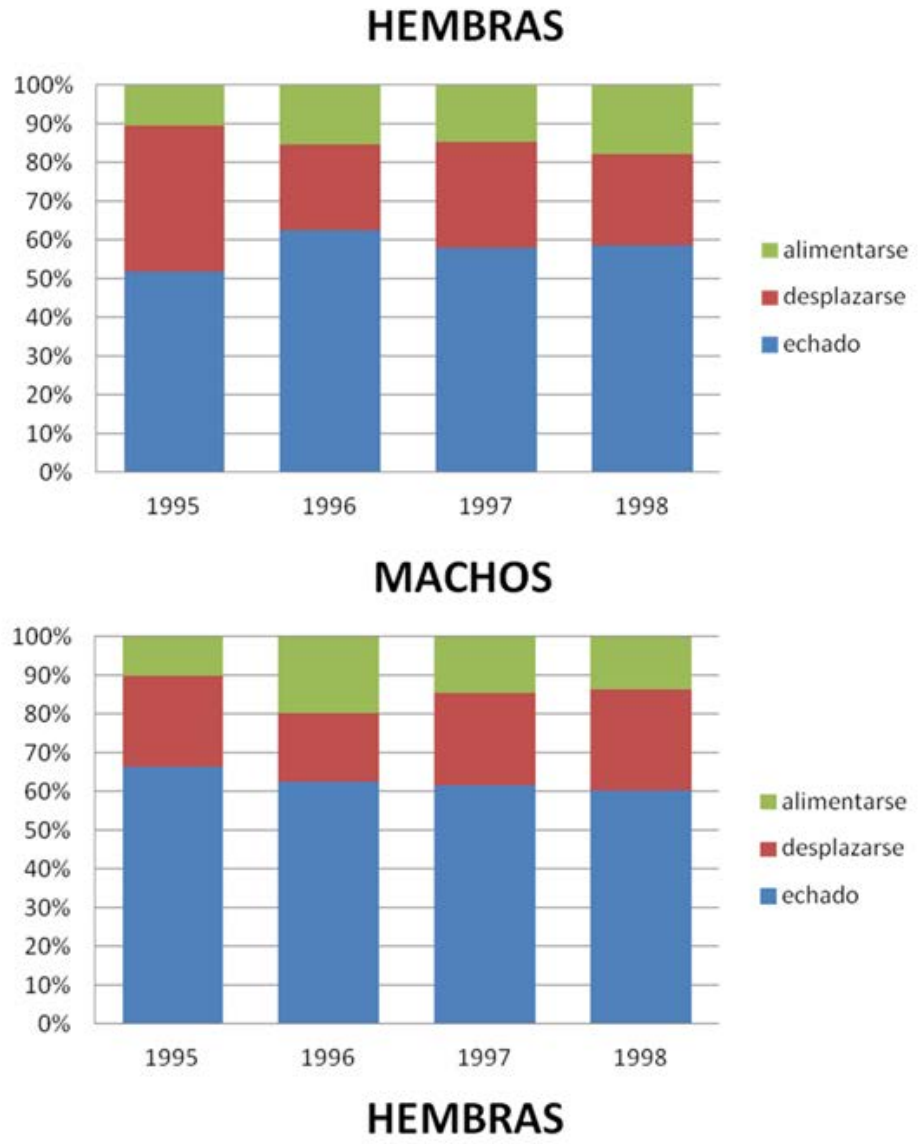

Figura 2. Patrón de actividad anual del venado cola blanca considerando el porcentaje dedicado las tres pautas de comportamiento durante los ciclos de $24 \mathrm{~h}$, de hembras y machos. 
Tabla 2. Resultados comparativos de la conducta de venados entre años y sexo, que tuvieron diferencias significativas $(P<0.05)$ considerando las pautas de comportamiento utilizando la Prueba Kruskal-Wallis y Comparaciones Múltiples de Mann-Whitney con el Programa PAST.

\begin{tabular}{lc}
\hline PAUTA DESPLAZARSE & VALORES DE SIGNIFICANCIA \\
\hline Hembras 1995-Hembras 1998 & 0.01927 \\
Hembras1998-Machos 1998 & 0.02527 \\
PAUTA ALIMENTARSE & \\
\hline Hembras 1997-Machos 1998 & 0.02960 \\
Machos 1995- Machos 1997 & 0.03118 \\
Machos 1997-Machos 1998 & 0.01455 \\
\hline
\end{tabular}

Alimentarse: No se encontraron diferencias entre los años entre las hembras, aunque 1998 hay un pico a las 07:00 h y en cuanto a los machos sí las hubo en 1995-1997 ( $P$ $=0.031)$, y 1997-1998 $(P=0.015)$, presentando la mayor frecuencia de 07:00 a 08:00 y de las 24:00 a la 01:00 h. Podemos señalar que sí hubo diferencias entre sexos entre hembras 1997 y el comportamiento de los machos en 1998 ( $P=0.030)$, que fue cuando ocurrió una distribución de las lluvias inusual, al verse atrasada la temporada de lluvias (Fig. 5).

\section{ECHADOS HEMBRAS}
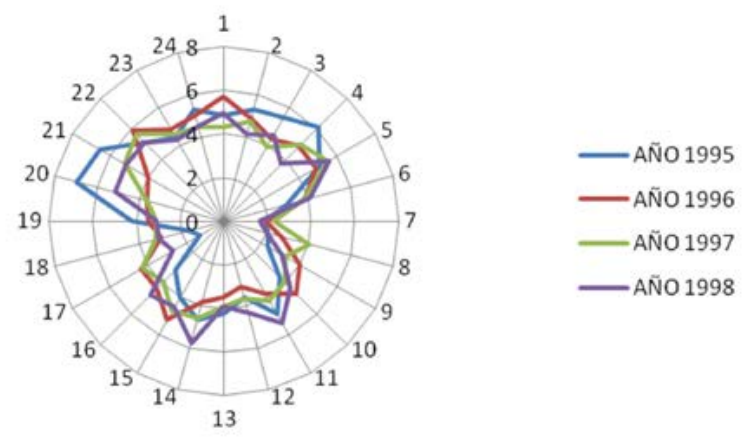

\section{ECHADOS MACHOS}

Figura 3. Porcentaje del tiempo que dedicaron los venados cola blanca a estar echado, distribuido en el ciclo de $24 \mathrm{~h}$, de hembras y machos.

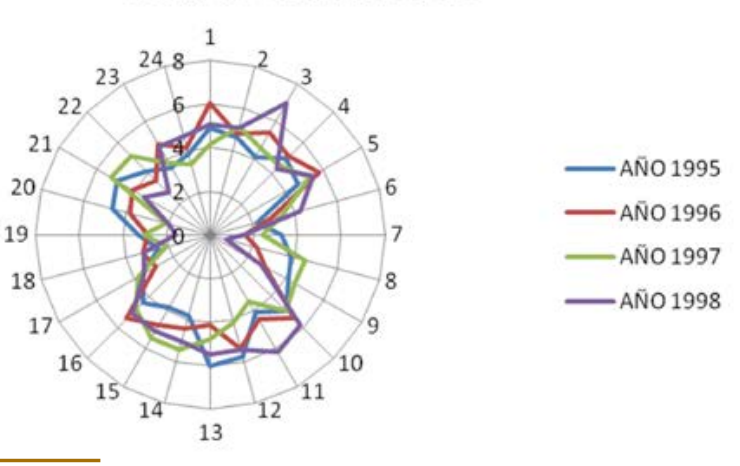

Al hacer una correlación de las pautas por sexo y la precipitación anual (Tabla 3), se encontró que hembras y machos responden de manera diferente a la precipitación, encontrando en hembras valores positivos altos en cuanto a la pauta de alimentación $(r=0.97)$ y valores negativos en cuanto al desplazamiento $(r=-0.85)$. Lo que significa que dedicaron más tiempo a alimentarse y se mueven menos en años con mayor precipitación, es decir cuando hay mayor cantidad de alimento, y en los machos en cuanto a la pauta de estar echados $(r=-0.92)$, o sea que descansan más en años con menor precipitación o condiciones de sequía más pronunciada. 


\section{Discusión}

En otros estudios, Beier y McCullough (1990) registraron que los venados cola blanca tuvieron patrones de actividad anuales diferentes entre los sexos, encontrando que las hembras están activas un mayor porcentaje del tiempo. También en nuestro estudio encontramos más activas a las hembras que a los machos, pero además, las primeras dedican más tiempo tanto a desplazarse como a alimentarse.

\section{MOVIMIENTO HEMBRAS}

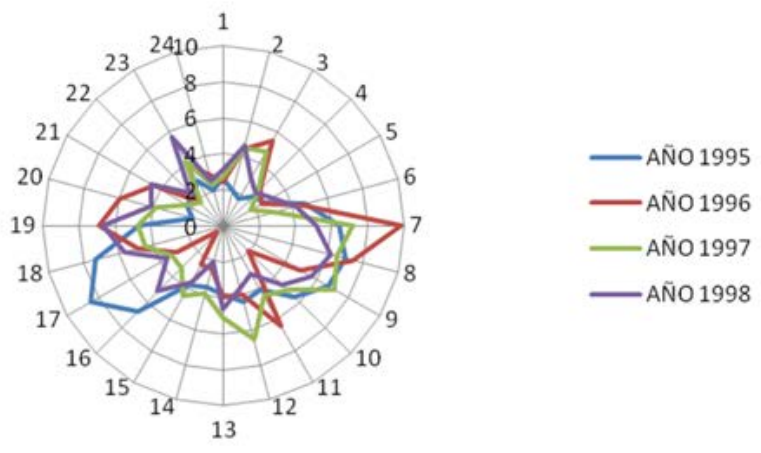

\section{MOVIMIENTO MACHOS}

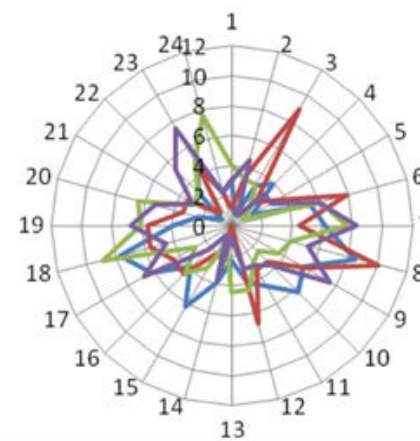

Figura 4. Porcentaje del tiempo que dedicaron los venados cola blanca a desplazarse o moverse, distribuido en el ciclo de $24 \mathrm{~h}$, de hembras y machos.

En general podemos decir que no hubo diferencias en el patrón general, pero si analizamos las pautas por separado, sólo encontramos diferencias significativas tanto en 1995 en algunas de las pautas como en el año 1998. Estos años fueron contrastantes en cuanto a la precipitación, ya que 1995 fue el año más seco del período de estudio y las Iluvias se presentaron en julio, pero en los siguientes meses la precipitación fue mínima. Mientras que en 1998 fue un año con mayor precipitación pero con un retraso en las Iluvias, afectando la supervivencia de las crías ya que la mayoría murieron al no tener alimento suficiente, tanto las hembras para la lactancia como las crías al destete.

Los patrones de actividad pueden estar reflejando cambios anuales en cuanto al tiempo que dedican los venados a alimentarse, para cubrir sus necesidades metabólicas ligadas a los cambios fisiológicos, enfrentando los cambios que ocurren en cuanto a la cantidad y calidad del forraje disponible, para poder mantener reservas energéticas. Las hembras incrementaron de un $10 \%$ (1995) a un $20 \%$ (1998) de su tiempo a forrajear. Aunque lo que sucedió en el año 1998 que se atrasaron las lluvias, tuvieron un efecto negativo en la población porque se alargó la época de sequía ocasionando que la mayoría de las crías se murieran. 
En la misma zona árida cuya vegetación es un matorral xerófilo, encontraron que el gasto energético para cada actividad (alimentación, desplazamiento y descanso) fue diferente (Gallina y Bello 2010). Estos resultados muestran que los machos en general gastan mayor energía que las hembras. Aunque cuando las condiciones de sequía son muy severas, ambos sexos presentan un menor gasto para alimentarse y moverse. Lo que indica que los venados de estas zonas tienen una estrategia conductual adaptada para tratar de ahorrar energía cuando las condiciones ambientales son desfavorables.

Las hembras consumen una dieta de mayor calidad que la que consumen los machos y, por lo tanto deben invertir más tiempo forrajeando para obtener alimento de mayor calidad para satisfacer sus requerimientos (Beier 1987). Los datos obtenidos demuestran que las hembras incrementaron el tiempo que dedican a alimentarse en años con mayor precipitación, cuando existe una mayor disponibilidad de alimento. Situación también registrada en la Reserva de la Biosfera Sierra de Huautla en Morelos, mediante el análisis del nitrógeno fecal. Las hembras consumieron alimento de mejor calidad proteica y utilizaron hábitats de mejor calidad de acuerdo a las características de la vegetación de los sitios, lo que demostró una segregación sexual en cuanto a este parámetro en la selva baja caducifolia (Gallina et al. 2014).

\section{ALIMENTACION HEMBRAS}

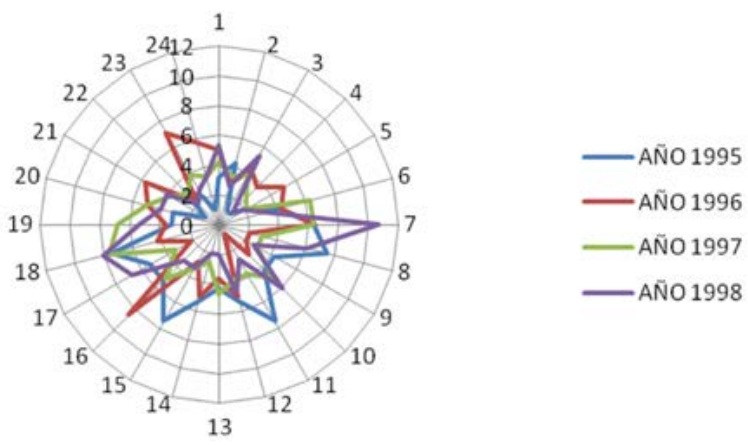

ALIMENTACION MACHOS

Figura 5. Porcentaje del tiempo que dedicaron los venados cola blanca a alimentarse, distribuido en el ciclo de $24 \mathrm{~h}$, de hembras y machos.

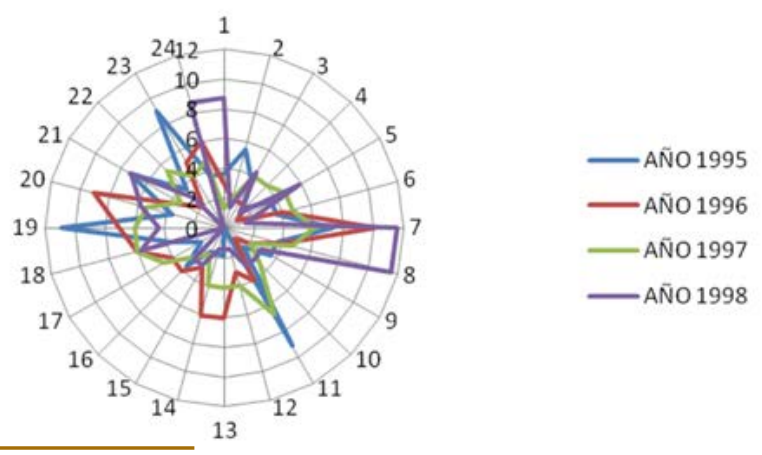

Se ha registrado que el comportamiento del venado cola blanca difiere según la época fisiológica (reproductiva, de gestación o postreproductiva y de crianza) y el sexo, dedicando el menor tiempo a alimentarse (11 - 16 \%) y el mayor a estar echados (57 - $64 \%$ respectivamente). La mayor actividad se presentó en la época reproductiva (que va de noviembre a febrero), cuando las temperaturas fueron menores, mientras que en el verano cuando la temperatura llega a más de $40{ }^{\circ} \mathrm{C}$, la actividad disminuye 
notoriamente (Gallina et al. 2005). Aunque en el presente estudio se hace un análisis anual (no separado por épocas fisiológicas), se mantiene la frecuencia similar de las actividades en el tiempo, aunque las hembras presentaron cambios significativos en algunas pautas como la alimentación.

Otros estudios sobre conducta de ungulados ramoneadores han mostrado que reducen su actividad e incrementan el descanso en días con elevadas temperaturas (du Toit y Yetman 2005), sugiriendo que los venados descansan durante el día, y se dedican a forrajear en la noche cuando las temperaturas son más bajas. En este trabajo se encontró que tanto hembras como machos presentan un pico a las 7:00 h, mientras que las hembras entre las 16:00 y 18:00 h también presentaron otro pico de actividad. Los machos forrajearon más durante las horas de la noche, mientras que las hembras descansaron durante ese período.

\begin{tabular}{|c|c|c|c|c|c|c|c|c|}
\hline AÑO & $\begin{array}{l}\text { Hembras- } \\
\text { Echado }\end{array}$ & $\begin{array}{l}\text { Hembras- } \\
\text { Movimiento }\end{array}$ & $\begin{array}{l}\text { Hembras- } \\
\text { Alimentarse }\end{array}$ & $\begin{array}{l}\text { Machos- } \\
\text { Echado }\end{array}$ & $\begin{array}{l}\text { Machos- } \\
\text { Movimiento }\end{array}$ & $\begin{array}{l}\text { Machos- } \\
\text { Alimentarse }\end{array}$ & $\begin{array}{l}\text { Precipitación } \\
\text { Anual (mm) }\end{array}$ & \multirow{7}{*}{$\begin{array}{l}\text { Tabla 3. Porcentaje } \\
\text { dedicado a cada pauta } \\
\text { de hembras y machos } \\
\text { en los diferentes años y } \\
\text { la precipitación anual, } \\
\text { y en la parte inferior del } \\
\text { cuadro el coeficiente de } \\
\text { correlación entre esas } \\
\text { variables: Hembras- } \\
\text { Echado (H-E), Hembras- } \\
\text { Movimiento (H-M), } \\
\text { Hembras-Alimentarse (H- } \\
\text { A), Machos-Echado (M-E), } \\
\text { Machos-Movimiento (M- } \\
\text { M), Machos-Alimentarse } \\
\text { (M-A) y la precipitación } \\
\text { anual (mm). }\end{array}$} \\
\hline 1995 & 51.98 & 37.67 & 10.35 & 66.48 & 23.50 & 10.02 & 135.5 & \\
\hline 1996 & 62.64 & 22.11 & 15.25 & 62.55 & 17.73 & 19.72 & 285.5 & \\
\hline 1997 & 56.44 & 25.59 & 17.97 & 61.49 & 23.87 & 14.64 & 368 & \\
\hline \multirow[t]{3}{*}{1998} & 58.57 & 23.43 & 18.00 & 60.23 & 26.06 & 13.71 & 318 & \\
\hline & $\mathrm{H}-\mathrm{E}$ & $\mathrm{H}-\mathrm{M}$ & $\mathrm{H}-\mathrm{A}$ & $M-E$ & $M-M$ & M-A & & \\
\hline & 0.57898491 & -0.85333159 & 0.97487957 & -0.92186998 & 0.08362861 & 0.54824928 & & \\
\hline
\end{tabular}

Durante los cuatro años, los venados presentaron desplazamientos en horas tempranas del día evitando las horas de mayor calor entre las 10:00 y 16:00 h para no perder agua por transpiración. Se encontró una correlación negativa significativa con la temperatura, entre el tiempo que los venados estaban activos a lo largo de los períodos del día (Gallina et al. 2005). Como ha sido señalado por otros autores, los venados reducen su actividad en los períodos del día con mayor estrés térmico (Drolet 1976; Beier y McCullough 1990). En Texas los venados fueron más activos en la mañana y en la tarde, pero este patrón de actividad cambió cuando existieron presiones de cacería o de actividades humanas (Jackson et al. 1972). En el área de estudio los venados no tuvieron esas presiones, ya que no había cacería ni actividades humanas, por lo que su comportamiento no se vio alterado.

En otras especies relacionadas con el venado cola blanca como el venado de las Pampas (Ozotoceros bezoarticus), se ha encontrado que tienen períodos de actividad tanto diurna como nocturna tanto en el Parque Nacional Emas como en los cerrados (las savanas) de Brasilia en el centro de Brasil (Leeuwenberg et al. 1997; Merino et al. 1997; Rodrigues y Monteiro-Filho 2000). En el ciervo rojo (Cervus elaphus) en Europa, la actividad diaria se ve influenciada por los ciclos de luz y obscuridad, presentando picos bimodales (Kamler et al. 2007). Lo mismo ocurre con otros cérvidos (el alce Alces alces y el corzo Capreolus capreolus) en Suecia, siendo más activos durante el amanecer y atardecer (Cederlund 1989). 
En este trabajo se encontró que los venados tuvieron un patrón de actividad crepuscular con picos de mayor actividad al amanecer y atardecer, como ha ocurrido con otros cérvidos, aunque al diferenciar las pautas de comportamiento, lo que no se ha hecho en otros estudios, sí llegan a modificar la proporción del tiempo que dedican a cada una de ellas. Los venados se adaptan a los cambios ambientales, sobre todo si se considera que la cantidad y calidad de alimento disponible depende de la cantidad y distribución de la precipitación (Bello et al. 2003). Además se encontró que las hembras y los machos pueden cambiar sus actividades dependiendo de la precipitación, respondiendo de manera diferente a las condiciones ambientales, siendo más sensibles las hembras, sobre todo por el gasto energético que representa la gestación y la lactancia. En algunos años pueden presentar diferencias significativas el porcentaje que dedican a cada pauta, así como la respuesta entre sexos, lo que muestra su adaptabilidad a factores ambientales tomando en cuenta que la precipitación es un factor crítico en las zonas áridas.

\section{Agradecimientos}

Al Consejo Nacional de Ciencia y Tecnología (CONACYT) que apoyó financieramente con el proyecto No. 225260-5-2480PB, a Ducks Unlimited de México (DUMAC) propietarios en ese entonces del Rancho San Francisco y brindaron todas las facilidades logísticas. A todos los que apoyaron para tomar los datos de campo: C. Delfin Alfonso, C. Contreras Verteramo, N. D. López, S. Lara, A. Pérez Arteaga, S. Mandujano y en especial a S. Ortíz, encargado del rancho por su valiosa ayuda. A B. Delfosse por la versión en inglés del Abstract. A los revisores que con sus comentarios mejoraron sustancialmente el manuscrito.

\section{Literatura citada}

BeIER, P. 1987. Sex differences in quality of white-tailed deer diets. Journal of Mammalogy 68:323-329.

Beier, P., y D. R. Mccullough. 1990. Factors influencing white-tailed deer activity patterns and habitat use. Wildlife Monographs 109:51.

Bello, J., S. Gallina, y M. Equinua. 2001A. Characterization and habitat preferences by white-tailed deer in Mexico with high drinking water availability. Journal of Range Management 54:537-545.

Bello, J., S. Gallina, M. Equinua, S. Mandujano, y C. Delfin. 2001B. Activity areas and distance to water sources by white-tailed deer in Northeastern Mexico. Vida silvestre Neotropical 10:30-37

Bello, J., S. Gallina, y M. Equinua. 2003A. El venado cola blanca: uso del hábitat en zonas semiáridas y con alta disponibilidad de agua del Noreste de México. Pp. 67-76 en Manejo de Fauna silvestre en Amazonía y Latinoamérica-Criterios de Sostenibilidad (Polanco-Ochoa, R., ed.). CITES, Fundación Natura. Bogotá, Colombia.

Bello, J., S. Gallina, y M. Equinua. 2003B. Comparación de los movimientos del venado cola blanca en dos sitios con diferente disponibilidad de agua del Noreste de México. Pp. 59-66 en Manejo de Fauna silvestre en Amazonía y Latinoaméricacriterios de sostenibilidad (Polanco-Ochoa, R., ed.). CITES, Fundación Natura. Bogotá, Colombia. 
Bello, J., S. Galuina, y M. Equinua. 2004. Movements of white-tailed deer and their relationship with the precipitation in the Northeastern of Mexico. Interciencia 29:357-361.

Briones, V. O. 1984. Sinecología y florística de Lampazos de Naranjo, Nuevo León (México), con énfasis en la Gran Llanura. Tesis Licenciatura, Universidad Autónoma de Nuevo León. Linares, México.

Cederlund, G. 1989. Activity patterns in moose and roe deer in a north boreal forest. Holarctic Ecology 12:39-45.

Corona-Zarate, P. 1999. Patrones de actividad del venado cola blanca (Odocoileus virginianus texanus Zimmerman, 1870). Tesis Licenciatura. Facultad de Biología. Universidad Veracruzana. Xalapa, México.

Drolet, C. A. 1976. Distribution and movements of white-tailed deer in southern New Brunswick in relation to environmental factors. Canadian Field Naturalist 90:123136.

Du Toit, J. T., y C. A. Yetman. 2005. Effects of body size on the diurnal activity budgets of African browsing ruminants. Oecologia 143:317-325.

Eberhardt, L. E., E. E. Hanson, y L. L. Cadwell. 1984. Movement and activity patterns of mule deer in the sagebrush-steppe region. Journal of Mammalogy 65:409-409.

Galuina, S., y J. Bello. 2010. El gasto energético del venado cola blanca (Odocoileus virginianus texanus) en relación a la precipitación en una zona semiárida de México. Therya 1:1-8

Galuina, S., A. Pérez-Arteaga, y S. Mandujano. 1998. Patrones de actividad del venado cola blanca (Odocoileus virginianus texanus) en un matorral xerófilo de México. Boletín Sociedad Biólogica. Concepción, Chile 69:221-228.

Gallina, S., P. Corona, y J. Bello. 2003. El venado cola blanca: comportamiento en zonas semiáridas del Noreste de México. Pp. 165-173 en Manejo de Fauna silvestre en Amazonía y Latinoamérica-Criterios de Sostenibilidad (Polanco-Ochoa, R., ed.). CITES, Fundación Natura. Bogotá Colombia.

Gallina, S., P. Corona, y J. Bello. 2005. El comportamiento del venado cola blanca en zonas semiáridas del Noreste de México. Pp. 193-204 en Contribuciones mastozoológicas en homenaje a Bernardo Villa (Sánchez-Cordero, V., y R. A. Medellín, eds.). Instituto de Biología- Instituto de Ecología, Universidad Nacional Autónoma de México, Comisión Nacional de la Biodiversidad. Ciudad de Mèxico, México.

Gallina, S., G. Sánchez-Rojas, A. Buenrostro-Silva, y C.a. López-González. 2014. Comparison of faecal nitrogen concentration between sexes of White-tailed Deer in a tropical dry forest in southern Mexico. Ethology Ecology and Evolution. DOI :10.1080/03949370.2014.891537

Halle, S., y S. N. Stenseth. 2000. Activity Patterns in Small Mammals. An Ecological Approach. Ecological Studies 141. Springer. New York, EE.UU.

Hammer, O., D. A. T. Harper, y P. D. Ryan. 2001. PAST: Paleontological Statistics software Package for Education and Data Analysis. Palaeontologia Electronica 4:9.

Jackson, R. M., M. White, y F. F. KnOwLton. 1972. Activity patterns of young white-tailed deer fawns in south Texas. Ecology 53:262-270. 
Kamler, J. F., B. JXdrzejewska, y W. JxDrZejewskı. 2007. Activity patterns of red deer in Białowieża National Park, Poland. Journal of Mammalogy 88:508-514.

Kroll, J. C. 1992. A practical guide to producing and harvesting white-tailed deer. Institute of white-tailed deer management and research center for applied studies in forestry. Stephen F. Austin State University. Austin, EE.UU.

Lariviere, S., J. Huot, y C. Samson. 1994. Daily activity patterns of female black bears in a northern mixed-forest environment. Journal of Mammalogy 75:613-620.

Leeumenberg, F., S. Lara-Resende, F. H. G. Rodrigues, y M. X. A. Bezerril. 1997. Home range,activity and habitat use of the Pampas deer (Ozotoceros bezoarticus L. 1758, Artiodactyla, Cervidae) in the Brazilian Cerrado. Mammalia 61:487-495.

Mandujano, S., S. Pérez, R. Sánchez, y S. Galuina. 1996. Diferenciación de pautas de actividad del venado con ayuda de radiotransmisores con sensor de movimiento. Acta Zoológica Mexicana (n. s.) 67:67-78

Merino, M. L., S. Gonzales, F. Leeumenberg, F. H.g. Rodrigues, L. Pinder, y W. M. Tomas. 1997. Veado campeiro (Ozotoceros bezoarticus Linnaeus 1758): distribuición, historia natural, ecología y conservación. Pp. 42-58 en Biologia y conservación de dos cervidos Sud-Americanos (Duarte, J. M. B., ed.). Fundación de Estudios e Investigaciones en Agronomía, Medicina Veterinaria y Zootecnia. Jaboticabal. Sao Paulo, Brasil.

Moen, A.N. 1978. Seasonal changes in heart rates, activity, metabolism, and forage intake of white-tailed deer. Journal of Wildlife Management 42:715-738,

Ockenfels, R. A., Y J. A. Bissonette. 1984. Temperature related responses in north-central Oklahoma white-tailed deer. Pp. 64-67 en Deer in the southwest: A workshop. (Krausman, P. R., y N. S. Smith, eds.). Arizona Cooperative Wildlife Research Unit, University of Arizona. Tucson, EE.UU.

Ockenfels, R. A., D. E. Brooks, y C. H. Lewis. 1991. General ecology of Coues whitetailed deer in the Santa Rita Mountains. Technical Report. Arizona Game and Fish Department. Phoenix, EE.UU.

Rivero, K., D. I. Rumiz, y A. B. Taber. 2005. Differential habitat use by two sympatric brocket deer species (Mazama americana and M. gouazoubira) in a seasonal Chiquitano forest of Bolivia. Mammalia 69:169-183.

Rodrigues, F. H. G., y E. L. A. Monteiro-Filho. 2000. Home range and activity patterns of Pampas deer in Emas National Park, Brazil. Journal of Mammalogy 81:11361142.

Rouleau, I., M.crete, y J. P. Ouellet. 2002. Contrasting the summer ecology of white tailed deer inhabiting a forested and an agricultural landscape. Ecoscience 9:459469

Verme, L. J., y D. E. Ulzrey. 1984. Physiology and nutrition. Pp. 91-118 en White-tailed deer: Ecology and Management (L. K. Halls, ed.). Published Stackpole Books. Harrisburg, EE.UU.

Wallmo, O. 1981. Mule and black-tailed deer of North America. A Wildlife Management Institute Book. Nebraska, EEUU.

Webb, S. L., M. R. Dzialak, D. Houchen, K. L. Kosciuch, y J. B. Winstead. 2013. Spatial ecology of female mule deer in an area proposed for wind energy development. Western North American Naturalist 73:347-356. 
Zar, J. H. 1984. Biostatistical Analysis. Second Edition. Prentice Hall. New Jersey, EE.UU.

Sometido: 6 de mayo de 2014

Revisado: 2 de Julio de 2014

Aceptado: 30 de julio de 2014

Editor asociado: Rafael Reyna

Diseño gráfico editorial: Gerardo Hernández 